\title{
Convergence theorems of modified Mann iterations
}

\author{
Jinzuo Chen and Dingping Wu*
}

"Correspondence:wdp68@163.com College of Applied Mathematics, Chengdu University of Information Technology, Xuefu Road, Chengdu, 610225, China

\begin{abstract}
In this paper, we introduce the modified iterations of Mann's type for nonexpansive mapping and asymptotically nonexpansive mapping to have the strong and weak convergence in a uniformly convex Banach space. We also proved strong convergence theorems of our modified Mann's iteration processes for nonexpansive semigroups and asymptotically nonexpansive semigroups. The results presented in the paper give a partially affirmative answer to the open question raised by Kim and Xu (Nonlinear Anal. 64:1140-1152, 2006). Applications to the accretive operators are also included.
\end{abstract}

MSC: 47H09; 47H10;47J25

Keywords: strong convergence; modified Mann iteration; uniformly convex Banach space; asymptotically nonexpansive mapping; nonexpansive semigroups; asymptotically nonexpansive semigroups; accretive operator

\section{Introduction}

Let $E$ be a real Banach space, $C$ a nonempty closed convex subset of $E$, and $T: C \rightarrow C$ a mapping. Recall that $T$ is a nonexpansive mapping [1] if $\|T x-T y\| \leq\|x-y\|$ for all $x, y \in C$, and $T$ is asymptotically nonexpansive [2] if there exists a sequence $\left\{k_{n}\right\}$ with $k_{n} \geq 1$ for all $n$ and $\lim _{n \rightarrow \infty} k_{n}=1$ and such that $\left\|T^{n} x-T^{n} y\right\| \leq k_{n}\|x-y\|$ for all integers $n \geq 1$ and $x, y \in C$. The class of asymptotically nonexpansive mappings was introduced by Goebel and Kirk [2] as an important generalization of the class of nonexpansive mappings, who proved that if $C$ is a nonempty closed convex subset of a real uniformly convex Banach space, and $T$ is an asymptotically nonexpansive mapping from $C$ into itself, then $T$ has a fixed point. A point $x \in C$ is a fixed point of $T$ provided $T x=x$. Denote by $\operatorname{Fix}(T)$ the set of fixed points of $T$; that is, $\operatorname{Fix}(T)=\{x \in C: T x=x\}$.

A family $\delta=\{T(t): t \geq 0\}$ is said to be an asymptotically nonexpansive semigroup [3] on $C$ with Lipschitzian constants $\left\{L_{t}: t>0\right\}$ if

(1) $t \mapsto L_{t}$ is a bounded, measurable, continuous mapping from $(0, \infty) \rightarrow[0, \infty)$;

(2) $\lim \sup _{t \rightarrow \infty} L_{t} \leq 1$;

(3) for each $t \geq 0, T(t)$ is a mapping from $C$ into itself, and $\|T(t) x-T(t) y\| \leq L_{t}\|x-y\|$ for each $x, y \in C$;

(4) $T(t+s) x=T(t) T(s) x$ for each $t, s \geq 0$ and $x \in C$;

(5) $T(0) x=x$ for each $x \in C$;

(6) for each $x \in C$, the mapping $t \mapsto T(t) x$ is continuous.

O2013 Chen and Wu; licensee Springer. This is an Open Access article distributed under the terms of the Creative Commons Attribution License (http://creativecommons.org/licenses/by/2.0), which permits unrestricted use, distribution, and reproduction in any medium, provided the original work is properly cited. 
8 is said to be nonexpansive semigroup on $C$ if $L_{t}=1$ for all $t>0$. We use Fix $(\delta)$ to denote the common fixed point set of the semigroup $\&$; that is, $\operatorname{Fix}(\delta)=\{x \in C: T(t) x=x, \forall t \geq 0\}$. Note that for an asymptotically nonexpansive semigroup $\Gamma$, we can always assume that the Lipschitzian constants $\left\{L_{t}\right\}_{t>0}$ are such that $L_{t} \geq 1$ for all $t>0$. $L$ is nonincreasing in $t$, and $\lim _{t \rightarrow \infty} L_{t}=1$; otherwise, we replace $L_{t}$ for each $t>0$, with $\widetilde{L}_{t}:=\max \left\{\sup _{s \geq t} L_{s}, 1\right\}$.

As is well known, the construction of fixed point of nonexpansive mappings and asymptotically nonexpansive mappings (and of common fixed points of nonexpansive semigroups and asymptotically nonexpansive semigroups) is an important subject in the theory of nonexpansive mappings, nonlinear operator theory and their applications: in particular, in image recovery, convex feasibility problem, convex minimization problem and signal processing problem [4-9].

Iterative approximation of a fixed point for nonexpansive mappings, asymptotically nonexpansive mappings, nonexpansive semigroups and asymptotically nonexpansive semigroups in Hilbert or Banach spaces including Mann [10], Ishikawa [11] and Halpern and Mann-type iteration algorithm [12] have been studied extensively by many authors to solve nonlinear operator equations as well as variational inequalities. However, the Mann iteration for nonexpansive mappings has in general only weak convergence even in a Hilbert space. More precisely, a Mann's iteration procedure is a sequence $\left\{x_{n}\right\}$, which is generated by

$$
x_{n+1}=\alpha_{n} x_{n}+\left(1-\alpha_{n}\right) T x_{n}, \quad n \geq 0,
$$

where the initial guess $x_{0} \in C$ is chosen arbitrarily. For example, Reich [13] proved that if $E$ is a uniformly convex Banach space with a Fréchet differentiable norm, and if $\left\{\alpha_{n}\right\}$ is chosen such that $\sum_{n=1}^{\infty} \alpha_{n}\left(1-\alpha_{n}\right)=\infty$, then the sequence $\left\{x_{n}\right\}$ defined by (1.1) converges weakly to a fixed point of $T$.

Some attempts to modify the Mann iteration method (1.1) so that strong convergence is guaranteed have recently been made. Nakajo and Takahashi [14] proposed the following modification of the Mann iteration method (1.1) for a nonexpansive mapping $T$ in a Hilbert space $H$ :

$$
\left\{\begin{array}{l}
x_{0} \in C \text { chosen arbitrarily, } \\
y_{n}=\alpha_{n} x_{n}+\left(1-\alpha_{n}\right) T x_{n}, \\
C_{n}=\left\{v \in C:\left\|y_{n}-v\right\| \leq\left\|x_{n}-v\right\|\right\}, \\
Q_{n}=\left\{v \in C:\left\langle x_{n}-v, x_{n}-x_{0}\right\rangle \leq 0\right\}, \\
x_{n+1}=P_{C_{n} \cap Q_{n}}\left(x_{0}\right),
\end{array}\right.
$$

where $P_{k}$ denotes the metric projection from $H$ onto a closed convex subset $K$ of $H$. They proved that if the $\left\{\alpha_{n}\right\}$ is bounded above from one, the sequence $\left\{x_{n}\right\}$ generated by (1.2) converges strongly to $P_{\operatorname{Fix}(T)}\left(x_{0}\right)$. Moreover, they introduced and studied an iteration pro- 
cess of a nonexpansive semigroup $\delta=\{T(t): t \geq 0\}$ in a Hilbert space $H$ :

$$
\left\{\begin{array}{l}
x_{0} \in C \text { chosen arbitrarily, } \\
y_{n}=\alpha_{n} x_{n}+\left(1-\alpha_{n}\right) \frac{1}{t_{n}} \int_{0}^{t_{n}} T(u) x_{n} d u, \\
C_{n}=\left\{v \in C:\left\|y_{n}-v\right\| \leq\left\|x_{n}-v\right\|\right\}, \\
Q_{n}=\left\{v \in C:\left\langle x_{n}-v, x_{n}-x_{0}\right\rangle \leq 0\right\}, \\
x_{n+1}=P_{C_{n} \cap Q_{n}}\left(x_{0}\right) .
\end{array}\right.
$$

Under the same condition of the sequence $\left\{\alpha_{n}\right\}$, and $\left\{t_{n}\right\}$ is positive real divergent sequence, the sequence $\left\{x_{n}\right\}$ generated by $(1.3)$ converges strongly to $P_{\mathrm{Fix}(T)}\left(x_{0}\right)$.

Kim and $\mathrm{Xu}$ [15], in 2006, adapted iteration (1.2) and (1.3) to asymptotically nonexpansive mapping and asymptotically nonexpansive semigroup. More precisely, they introduced the following iteration processes for asymptotically nonexpansive mapping $T$ and asymptotically nonexpansive semigroup $\delta=\{T(t): t \geq 0\}$, respectively, with $C$ a closed convex bounded subset of a Hilbert space $H$ :

$$
\left\{\begin{array}{l}
x_{0} \in C \text { chosen arbitrarily, } \\
y_{n}=\alpha_{n} x_{n}+\left(1-\alpha_{n}\right) T^{n} x_{n}, \\
C_{n}=\left\{z \in C:\left\|y_{n}-v\right\|^{2} \leq\left\|x_{n}-v\right\|^{2}+\theta_{n}\right\}, \\
Q_{n}=\left\{z \in C:\left\langle x_{n}-z, x_{0}-x_{n}\right\rangle \geq 0\right\}, \\
x_{n+1}=P_{C_{n} \cap Q_{n}}\left(x_{0}\right),
\end{array}\right.
$$

where $\theta_{n}=\left(1-\alpha_{n}\right)\left(k_{n}^{2}-1\right)(\operatorname{diam} C)^{2} \rightarrow 0$ as $n \rightarrow \infty$ and

$$
\left\{\begin{array}{l}
x_{0} \in C \text { chosen arbitrarily, } \\
y_{n}=\alpha_{n} x_{n}+\left(1-\alpha_{n}\right) \frac{1}{t_{n}} \int_{0}^{t_{n}} T(u) x_{n} d u, \\
C_{n}=\left\{z \in C:\left\|y_{n}-v\right\|^{2} \leq\left\|x_{n}-v\right\|^{2}+\tilde{\theta}_{n}\right\}, \\
Q_{n}=\left\{z \in C:\left\langle x_{n}-z, x_{0}-x_{n}\right\rangle \geq 0\right\}, \\
x_{n+1}=P_{C_{n} \cap Q_{n}}\left(x_{0}\right),
\end{array}\right.
$$

where $\tilde{\theta}_{n}=\left(1-\alpha_{n}\right)\left[\left(\frac{1}{t_{n}} \int_{0}^{t_{n}} L_{s} d s\right)^{2}-1\right](\operatorname{diam} C)^{2} \rightarrow 0$ as $n \rightarrow \infty$.

They proved that both iteration processes (1.4) and (1.5) converge strongly to a fixed point of $T$ and a common fixed point of $\&$, respectively, provided $\alpha_{n} \leq a$ for all integers $n, 0<a<1$ and $\left\{t_{n}\right\}$ is a positive real divergent sequence, using the boundedness of the closed convex subset of $C$ and Lipschitzian constant $L_{t}$ of the mapping $T(t)$.

Without knowing the rate of convergence of (1.2), Kim and Xu [16] in 2005, proposed a simpler modification of Mann's iteration method (1.1) for a nonexpansive mapping $T$ in a uniformly smooth Banach space $E$,

$$
\left\{\begin{array}{l}
x_{0} \in C \text { chosen arbitrarily, } \\
y_{n}=\alpha_{n} x_{n}+\left(1-\alpha_{n}\right) T x_{n}, \\
x_{n+1}=\beta_{n} u+\left(1-\beta_{n}\right) y_{n},
\end{array}\right.
$$


where $u \in C$ is an arbitrary fixed point element in $C$. They proved that $\left\{\alpha_{n}\right\}$ and $\left\{\beta_{n}\right\}$ are two sequences in $(0,1)$, satisfying certain assumptions, then $\left\{x_{n}\right\}$ defined by $(1.6)$ converges to a fixed point of $T$.

In [15], Kim and Xu adapted iteration (1.2) and (1.3) to asymptotically nonexpansive mappings and asymptotically nonexpansive semigroups. At the same time, they also raised the following open question.

Open question [15] Apparently, the iteration method (1.6) is simpler than (1.2). However, we do not know if we can adapt the method (1.6) to asymptotically nonexpansive mappings and asymptotically nonexpansive semigroups.

It is the purpose of this paper to develop iteration (1.6) to the processes for nonexpansive mappings, asymptotically nonexpansive mappings, nonexpansive semigroups and asymptotically nonexpansive semigroups in the frame of uniformly convex Banach space in Section 3 and Section 4. More precisely, we introduce the following modified Mann iteration processes for nonexpansive mappings, asymptotically nonexpansive mappings $T$ and nonexpansive semigroups, asymptotically nonexpansive semigroups $\delta=\{T(t): t \geq 0\}$, respectively, with $C$ a closed convex subset of a Banach space $E$ :

$$
\left\{\begin{array}{l}
x_{0} \in C \text { chosen arbitrarily, } \\
y_{n}=\alpha_{n} x_{n}+\left(1-\alpha_{n}\right) T^{n} x_{n}, \\
x_{n+1}=\beta_{n} u+\left(1-\beta_{n}\right) y_{n}, \quad n \geq 0
\end{array}\right.
$$

and

$$
\left\{\begin{array}{l}
x_{0} \in C \text { chosen arbitrarily, } \\
y_{n}=\alpha_{n} x_{n}+\left(1-\alpha_{n}\right) \frac{1}{t_{n}} \int_{0}^{t_{n}} T(u) x_{n} d u, \\
x_{n+1}=\beta_{n} u+\left(1-\beta_{n}\right) y_{n}, \quad n \geq 0 .
\end{array}\right.
$$

The strong and weak convergence of the sequence $\left\{x_{n}\right\}$ to a fixed point of nonexpansive mappings, asymptotically nonexpansive mappings $T$ are established. Strong convergence theorems for nonexpansive semigroups and asymptotically nonexpansive semigroups $\delta=$ $\{T(t): t \geq 0\}$ are also obtained. Therefore, results presented in the paper give a partially affirmative answer to the open question raised by Kim and $\mathrm{Xu}$ [15].

Our second modification of Mann's iteration method (1.1) is adaption to (1.6) for finding a zero of an $m$-accretive operator $A$, for which we assume that the zero set $A^{-1}(0) \neq \emptyset$. Our iterations process $\left\{x_{n}\right\}$ is given by

$$
\left\{\begin{array}{l}
x_{0} \in E \text { chosen arbitrarily, } \\
y_{n}=\alpha_{n} x_{n}+\left(1-\alpha_{n}\right) J_{r_{n}} x_{n}, \\
x_{n+1}=\beta_{n} u+\left(1-\beta_{n}\right) y_{n}, \quad n \geq 0
\end{array}\right.
$$

and another sequence $\left\{x_{n}\right\}$ as follows:

$$
\left\{\begin{array}{l}
x_{0} \in E \text { chosen arbitrarily, } \\
y_{n}=\alpha_{n} x_{n}+\left(1-\alpha_{n}\right) J_{r_{1, n}} x_{n}, \\
x_{n+1}=\beta_{n} J_{r_{1, n}} x_{n}+\left(1-\beta_{n}\right) J_{r_{2, n}} y_{n}, \quad n \geq 0 .
\end{array}\right.
$$


where for each $r>0, J_{r}=(I+r A)^{-1}$ is the resolvent of $A$. We prove that only in a uniformly convex Banach space and under certain appropriate assumptions on the sequences $\left\{\alpha_{n}\right\}$, $\left\{\beta_{n}\right\}$ and which will be made precise in Section 5 that $\left\{x_{n}\right\}_{n=0}^{\infty}$ defined by (1.9) and (1.10) converge strongly to a zero of $A$.

We write $x_{n} \rightarrow x$ to indicate that the sequence $\left\{x_{n}\right\}$ converges weakly to $x$. Similarly, $x_{n} \rightarrow x$ will symbolize strong convergence.

\section{Preliminaries}

This section collects some lemmas, which will be used in the proofs for the main results in the next section.

Lemma 2.1 [17] Let $\left\{a_{n}\right\},\left\{b_{n}\right\}$ and $\left\{\delta_{n}\right\}$ be sequences of nonnegative real numbers satisfying the inequality

$$
a_{n+1} \leq\left(1+\delta_{n}\right) a_{n}+b_{n}, \quad n \geq 1 .
$$

If $\sum_{n=1}^{\infty} \delta_{n}<\infty$ and $\sum_{n=1}^{\infty} b_{n}<\infty$, then

(1) $\lim _{n \rightarrow \infty} a_{n}$ exists;

(2) $\lim _{n \rightarrow \infty} a_{n}=0$ whenever $\liminf _{n \rightarrow \infty} a_{n}=0$.

Lemma 2.2 [18] Suppose that $E$ is a uniformly convex Banach space, and $0<t_{n}<1$ for all $n \in N$. Let $\left\{x_{n}\right\}$ and $\left\{y_{n}\right\}$ be two sequences of $E$ such that $\lim _{\sup _{n \rightarrow \infty}}\left\|x_{n}\right\| \leq r$, limsup $\sup _{n \rightarrow \infty}\left\|y_{n}\right\| \leq r$ and $\lim _{n \rightarrow \infty}\left\|t_{n} x_{n}+\left(1-t_{n}\right) y_{n}\right\|=r$ hold for some $r \geq 0$, then $\lim _{n \rightarrow \infty}\left\|x_{n}-y_{n}\right\|=0$.

Lemma 2.3 [19] Let C be a nonempty closed convex subset of a uniformly convex Banach space $E$, and $T: C \rightarrow C$ be an asymptotically nonexpansive mapping. Then $I-T$ is demiclosed at zero, i.e., if $x_{n} \rightarrow x$ and $x_{n}-T x_{n} \rightarrow x$, then $x \in \operatorname{Fix}(T)$.

Lemma 2.4 [20] A real Banach space $E$ is said to satisfy Opial's condition if the condition $x_{n} \rightarrow x$ implies

$$
\limsup _{n \rightarrow \infty}\left\|x_{n}-x\right\|<\limsup _{n \rightarrow \infty}\left\|x_{n}-y\right\|,
$$

for all $x \neq y, x, y \in E$.

Lemma 2.5 [21] A mapping $T: C \rightarrow C$ with a nonempty fixed point set $F$ in $C$ will be said to satisfy Condition (I):

If there is a nondecreasing function $f:[0, \infty) \rightarrow[0, \infty)$ with $f(0)=0, f(r)>0$ for all $r \in(0, \infty)$ such that $\|x-T x\| \geq f(d(x, F))$ for all $x \in C$, where $d(x, F)=\inf \{\|x-p\|: p \in F\}$.

Lemma 2.6 [22] Let $C$ be a nonempty closed convex subset of a uniformly convex Banach space $E, D$ a bounded closed convex subset of $C$ and $s=\{T(t): t \geq 0\}$ a nonexpansive semigroup (asymptotically nonexpansive semigroup) on $C$, such that $\operatorname{Fix}(\delta) \neq \emptyset$. For each $h \geq 0$, then

$$
\lim _{t \rightarrow \infty} \sup _{x \in D}\left\|\frac{1}{t} \int_{0}^{t} T(u) x d u-T(h) \frac{1}{t} \int_{0}^{t} T(u) x d u\right\|=0 .
$$


Lemma 2.7 [23] For $\lambda>0$ and $\mu>0$ and $x \in E$, the following identity holds

$$
J_{\lambda} x=J_{\mu}\left(\frac{\mu}{\lambda}+\left(1-\frac{\mu}{\lambda}\right) J_{\lambda} x\right)
$$

\section{Convergence to a fixed point of nonexpansive mapping and asymptotically nonexpansive mapping}

In this section, we prove weak and strong convergence theorems for asymptotically nonexpansive mappings and strong convergence theorem for nonexpansive mappings.

Theorem 3.1 Let $C$ be a nonempty closed convex subset of a uniformly convex Banach space $E$, and let $T: C \rightarrow C$ be a nonexpansive mapping satisfying Condition (I) and $\operatorname{Fix}(T) \neq \emptyset$. Given a point $u \in C$, and given that $\left\{\alpha_{n}\right\}$ and $\left\{\beta_{n}\right\}$ are two sequences in $(0,1)$ such that $\sum \beta_{n}<\infty$.

Define a sequence $\left\{x_{n}\right\}_{n=0}^{\infty}$ in C by algorithm (1.6), then $\left\{x_{n}\right\}_{n=0}^{\infty}$ strongly converges to a fixed point of $T$.

Proof First, we observe that $\left\{x_{n}\right\}$ is bounded, if we take an arbitrary fixed point $q$ of $F(T)$, noting that

$$
\begin{aligned}
\left\|y_{n}-q\right\| & =\left\|\alpha_{n} x_{n}+\left(1-\alpha_{n}\right) T x_{n}-q\right\| \\
& \leq \alpha_{n}\left\|x_{n}-q\right\|+\left(1-\alpha_{n}\right)\left\|T x_{n}-q\right\| \\
& \leq\left\|x_{n}-q\right\|,
\end{aligned}
$$

we have

$$
\begin{aligned}
\left\|x_{n+1}-q\right\| & =\left\|\beta_{n} u+\left(1-\beta_{n}\right) y_{n}-q\right\| \\
& \leq \beta_{n}\left\|u-y_{n}\right\|+\left\|y_{n}-q\right\| \\
& \leq \beta_{n}\|u-q\|+\beta_{n}\left\|y_{n}-q\right\|+\left\|y_{n}-q\right\| \\
& \leq\left(1+\beta_{n}\right)\left\|x_{n}-q\right\|+\beta_{n}\|u-q\| .
\end{aligned}
$$

By Lemma 2.1 and $\sum \beta_{n}<\infty$, thus, $\lim _{n \rightarrow \infty}\left\|x_{n}-q\right\|$ exists. Denote

$$
\lim _{n \rightarrow \infty}\left\|x_{n}-q\right\|=c
$$

Hence, $\left\{x_{n}\right\}$ is bounded, so is $\left\{y_{n}\right\}$. Now

$$
\begin{aligned}
\left\|x_{n+1}-q\right\| & =\left\|\beta_{n} u+\left(1-\beta_{n}\right) y_{n}-q\right\| \\
& =\left\|\beta_{n}\left(u-y_{n}\right)+\left(y_{n}-q\right)\right\| \\
& \leq \beta_{n}\left\|u-y_{n}\right\|+\left\|y_{n}-q\right\| .
\end{aligned}
$$

By $\sum \beta_{n}<\infty$, we obtain

$$
\lim _{n \rightarrow \infty}\left\|x_{n}-q\right\| \leq \liminf _{n \rightarrow \infty}\left\|y_{n}-q\right\|
$$


Since $\left\|y_{n}-q\right\| \leq\left\|x_{n}-q\right\|$, which implies that

$$
\limsup _{n \rightarrow \infty}\left\|y_{n}-q\right\| \leq \lim _{n \rightarrow \infty}\left\|x_{n}-q\right\|
$$

so that (3.2) and (3.3) give

$$
\lim _{n \rightarrow \infty}\left\|y_{n}-q\right\|=\lim _{n \rightarrow \infty}\left\|x_{n}-q\right\|=c .
$$

Moreover, $\left\|T x_{n}-q\right\| \leq\left\|x_{n}-q\right\|$ implies that

$$
\limsup _{n \rightarrow \infty}\left\|T x_{n}-q\right\| \leq c
$$

Thus,

$$
\begin{aligned}
c & =\lim _{n \rightarrow \infty}\left\|y_{n}-q\right\|=\lim _{n \rightarrow \infty}\left\|\alpha_{n} x_{n}+\left(1-\alpha_{n}\right) T x_{n}-q\right\| \\
& =\lim _{n \rightarrow \infty}\left\|\alpha_{n}\left(x_{n}-q\right)+\left(1-\alpha_{n}\right)\left(T x_{n}-q\right)\right\|,
\end{aligned}
$$

given by Lemma 2.2 that

$$
\lim _{n \rightarrow \infty}\left\|T x_{n}-x_{n}\right\|=0 .
$$

By (3.1) and $\sum \beta_{n}<\infty$, then we have

$$
\begin{aligned}
\left\|x_{n+m}-q\right\| & \leq\left(1+\beta_{n+m-1}\right)\left\|x_{n+m-1}-q\right\|+s_{n+m-1} \\
& \leq \mathrm{e}^{\beta_{n+m-1}}\left\|x_{n+m-1}-q\right\|+s_{n+m-1} \\
& \leq \mathrm{e}^{\beta_{n+m-1}} \mathrm{e}^{\beta_{n+m-2}}\left\|x_{n+m-2}-q\right\|+\mathrm{e}^{\beta_{n+m-1}} s_{n+m-2}+s_{n+m-1} \\
& \leq \mathrm{e}^{\beta_{n+m-1}+\beta_{n+m-2}}\left\|x_{n+m-2}-q\right\|+\mathrm{e}^{\beta_{n+m-1}}\left(s_{n+m-1}+s_{n+m-2}\right) \\
& \leq \cdots \\
& \leq \mathrm{e}^{\sum_{i=n}^{n+m-1} \beta_{i}}\left\|x_{n}-q\right\|+\mathrm{e}^{\sum_{i=n}^{n+m-1} \beta_{i}} \sum_{i=n}^{n+m-1} s_{i} .
\end{aligned}
$$

That is,

$$
\left\|x_{n+m}-q\right\| \leq M\left(\left\|x_{n}-q\right\|+\sum_{i=n}^{\infty} s_{i}\right),
$$

where $M=\mathrm{e}^{\sum_{i=n}^{n+m-1} \beta_{i}}$ for all $m, n \geq 1$, for all $q \in \operatorname{Fix}(T)$ and for $M>0$ and $s_{i}=\beta_{i}\|u-q\|$.

Next, we prove that $\left\{x_{n}\right\}_{n=0}^{\infty}$ is a Cauchy sequence.

Since $q \in \operatorname{Fix}(T)$ arbitrarily, and $\lim _{n \rightarrow \infty}\left\|x_{n}-q\right\|$ exists, consequently, $d\left(x_{n}, F\right)$ exists by Lemma 2.5. From Lemma 2.5 and (3.4), we get

$$
\lim _{n \rightarrow \infty} f\left(d\left(x_{n}, F\right)\right) \leq \lim _{n \rightarrow \infty}\left\|x_{n}-T x_{n}\right\|=0 .
$$


Since $f:[0, \infty) \rightarrow[0, \infty)$ is a nondecreasing function satisfying $f(0)=0, f(r)>0$ for all $r \in(0, \infty)$, therefore, we have

$$
\lim _{n \rightarrow \infty} d\left(x_{n}, F\right)=0 .
$$

Let $\varepsilon>0$, since $\lim _{n \rightarrow \infty} d\left(x_{n}, F\right)=0$ and $\sum_{i=0}^{\infty} s_{i}<\infty$, therefore, there exists a constant $n_{0}$ such that for all $n \geq n_{0}$, we have

$$
d\left(x_{n}, F\right) \leq \frac{\varepsilon}{3 M} \quad \text { and } \quad \sum_{j=n_{0}}^{\infty} s_{j} \leq \frac{\varepsilon}{6 M}
$$

in particular,

$$
d\left(x_{n_{0}}, F\right) \leq \frac{\varepsilon}{3 M} .
$$

There must exist $p_{1} \in \operatorname{Fix}(T)$, such that

$$
d\left(x_{n_{0}}, p_{1}\right) \leq \frac{\varepsilon}{3 M} .
$$

From (3.5), it can be obtained that when $n \geq n_{0}$,

$$
\begin{aligned}
\left\|x_{n+m}-x_{n}\right\| & \leq\left\|x_{n+m}-p_{1}\right\|+\left\|x_{n}-p_{1}\right\| \\
& \leq 2 M\left(\left\|x_{n_{0}}-p_{1}\right\|+\sum_{j=n_{0}}^{n_{0}+m-1} s_{j}\right) \\
& \leq 2 M\left(\frac{\varepsilon}{3 M}+\frac{\varepsilon}{6 M}\right)=\varepsilon .
\end{aligned}
$$

This implies that $\left\{x_{n}\right\}_{n=0}^{\infty}$ is a Cauchy sequence in a closed convex subset $C$ of a Banach space $E$. Thus, it must converge to a point in $C$, let $\lim _{n \rightarrow \infty} x_{n}=p$.

For all $\epsilon>0$, as $\lim _{n \rightarrow \infty} x_{n}=p$, thus, there exists a number $n_{1}$ such that when $n_{2} \geq n_{1}$,

$$
\left\|x_{n_{2}}-p\right\| \leq \frac{\epsilon}{4}
$$

In fact, $\lim _{n \rightarrow \infty} d\left(x_{n}, F\right)=0$ implies that using number $n_{2}$ above, when $n \geq n_{2}$, we have $d\left(x_{n}, F\right) \leq \frac{\epsilon}{8}$. In particular, $d\left(x_{n_{2}}, F\right) \leq \frac{\epsilon}{8}$. Thus, there must exist $\bar{p} \in F$, such that

$$
\left\|x_{n_{2}}-\bar{p}\right\|=d\left(x_{n_{2}}, \bar{p}\right)=\frac{\epsilon}{8} .
$$

From (3.6) and (3.7), we get

$$
\begin{aligned}
\|T p-p\| & =\left\|T p-\bar{p}+T x_{n_{2}}-\bar{p}+\bar{p}-x_{n_{2}}+x_{n_{2}}-p+\bar{p}-T x_{n_{2}}\right\| \\
& \leq\|T p-\bar{p}\|+\left\|x_{n_{2}}-\bar{p}\right\|+\left\|x_{n_{2}}-p\right\|+2\left\|T x_{n_{2}}-\bar{p}\right\| \\
& \leq\|p-\bar{p}\|+3\left\|x_{n_{2}}-\bar{p}\right\|+\left\|x_{n_{2}}-p\right\| \\
& \leq\left\|x_{n_{2}}-p\right\|+\left\|x_{n_{2}}-\bar{p}\right\|+3\left\|x_{n_{2}}-\bar{p}\right\|+\left\|x_{n_{2}}-p\right\|
\end{aligned}
$$




$$
\begin{aligned}
& =4\left\|x_{n_{2}}-\bar{p}\right\|+2\left\|x_{n_{2}}-p\right\| \\
& \leq \frac{4 \epsilon}{8}+\frac{2 \epsilon}{4}=\epsilon .
\end{aligned}
$$

As $\epsilon$ is an arbitrary positive number, thus, $T p=p$, so $\left\{x_{n}\right\}_{n=0}^{\infty}$ converges strongly to a point of $T$.

Theorem 3.2 Let $C$ be a nonempty closed convex subset of a uniformly convex Banach space $E$, and let $T: C \rightarrow C$ be an asymptotically nonexpansive mapping satisfying Condition (I) and $\operatorname{Fix}(T) \neq \emptyset$. Given a point $u \in C$, and given that $\left\{\alpha_{n}\right\}$ and $\left\{\beta_{n}\right\}$ are two sequences in $(0,1)$, the following conditions are satisfied:

(i) $\sum \beta_{n}<\infty$;

(ii) $\sum\left(k_{n}-1\right)<\infty$.

Define a sequence $\left\{x_{n}\right\}_{n=0}^{\infty}$ in C by algorithm (1.7), then $\left\{x_{n}\right\}_{n=0}^{\infty}$ strongly converges to a fixed point of $T$.

Proof First, we observe that $\left\{x_{n}\right\}$ is bounded, if we take an arbitrary fixed point $q$ of Fix $(T)$, noting that

$$
\begin{aligned}
\left\|y_{n}-q\right\| & =\left\|\alpha_{n} x_{n}+\left(1-\alpha_{n}\right) T^{n} x_{n}-q\right\| \\
& \leq \alpha_{n}\left\|x_{n}-q\right\|+\left(1-\alpha_{n}\right)\left\|T^{n} x_{n}-q\right\| \\
& =\left[\alpha_{n}+k_{n}\left(1-\alpha_{n}\right)\right]\left\|x_{n}-q\right\| \\
& \leq k_{n}\left\|x_{n}-q\right\|,
\end{aligned}
$$

we have

$$
\begin{aligned}
\left\|x_{n+1}-q\right\| & =\left\|\beta_{n} u+\left(1-\beta_{n}\right) y_{n}-q\right\| \\
& =\left\|\beta_{n}\left(u-y_{n}\right)+\left(y_{n}-q\right)\right\| \\
& \leq \beta_{n}\left\|u-y_{n}\right\|+k_{n}\left\|x_{n}-q\right\| \\
& =\beta_{n}\left\|u-q+q-y_{n}\right\|+k_{n}\left\|x_{n}-q\right\| \\
& \leq \beta_{n}\left\|y_{n}-q\right\|+\beta_{n}\|u-q\|+k_{n}\left\|x_{n}-q\right\| \\
& \leq \beta_{n} k_{n}\left\|x_{n}-q\right\|+k_{n}\left\|x_{n}-q\right\|+\beta_{n}\|u-q\| \\
& =\left[1+\left(\beta_{n} k_{n}+k_{n}-1\right)\right]\left\|x_{n}-q\right\|+\beta_{n}\|u-q\| .
\end{aligned}
$$

Put

$$
k_{\infty}=\sup \left\{k_{n}: n \geq 1\right\}<\infty .
$$

Thus, sequence $\left\{k_{n}\right\}$ is bounded, by Lemma 2.1 and Conditions (i), (ii), thus, $\lim _{n \rightarrow \infty} \| x_{n}-$ $q \|$ exists. Denote

$$
\lim _{n \rightarrow \infty}\left\|x_{n}-q\right\|=c
$$


Hence, $\left\{x_{n}\right\}$ is bounded, so is $\left\{y_{n}\right\}$. Now

$$
\begin{aligned}
\left\|x_{n+1}-q\right\| & =\left\|\beta_{n} u+\left(1-\beta_{n}\right) y_{n}-q\right\| \\
& =\left\|\beta_{n}\left(u-y_{n}\right)+\left(y_{n}-q\right)\right\| \\
& \leq \beta_{n}\left\|u-y_{n}\right\|+\left\|y_{n}-q\right\| .
\end{aligned}
$$

By assumption (i), we obtain $\lim _{n \rightarrow \infty}\left\|x_{n}-q\right\| \leq \liminf _{n \rightarrow \infty}\left\|y_{n}-q\right\|$. Since $\left\|y_{n}-q\right\| \leq$ $k_{n}\left\|x_{n}-q\right\|$, which implies that

$$
\limsup _{n \rightarrow \infty}\left\|y_{n}-q\right\| \leq \lim _{n \rightarrow \infty}\left\|x_{n}-q\right\|
$$

so that gives

$$
\lim _{n \rightarrow \infty}\left\|y_{n}-q\right\|=\lim _{n \rightarrow \infty}\left\|x_{n}-q\right\|=c .
$$

Moreover, $\left\|T^{n} x_{n}-q\right\| \leq k_{n}\left\|x_{n}-q\right\|$ implies that

$$
\limsup _{n \rightarrow \infty}\left\|T^{n} x_{n}-q\right\| \leq \lim _{n \rightarrow \infty}\left\|x_{n}-q\right\|=c .
$$

Thus,

$$
\begin{aligned}
c & =\lim _{n \rightarrow \infty}\left\|y_{n}-q\right\|=\lim _{n \rightarrow \infty}\left\|\alpha_{n} x_{n}+\left(1-\alpha_{n}\right) T^{n} x_{n}-q\right\| \\
& =\lim _{n \rightarrow \infty}\left\|\alpha_{n}\left(x_{n}-q\right)+\left(1-\alpha_{n}\right)\left(T^{n} x_{n}-q\right)\right\|,
\end{aligned}
$$

given by Lemma 2.2 that

$$
\lim _{n \rightarrow \infty}\left\|T^{n} x_{n}-x_{n}\right\|=0
$$

Now,

$$
\begin{aligned}
\left\|y_{n}-x_{n}\right\| & =\left\|\alpha_{n} x_{n}+\left(1-\alpha_{n}\right) T^{n} x_{n}-x_{n}\right\| \\
& \leq\left(1-\alpha_{n}\right)\left\|\left(T^{n} x_{n}-x_{n}\right)\right\| .
\end{aligned}
$$

Hence, by (3.9),

$$
\lim _{n \rightarrow \infty}\left\|y_{n}-x_{n}\right\|=0
$$

Also note that

$$
\begin{aligned}
\left\|x_{n+1}-x_{n}\right\| & =\left\|\beta_{n} u+\left(1-\beta_{n}\right) y_{n}-x_{n}\right\| \\
& \leq \beta_{n}\left\|u-x_{n}\right\|+\left(1-\beta_{n}\right)\left\|y_{n}-x_{n}\right\|,
\end{aligned}
$$

so that Condition (i) and (3.10) give

$$
\lim _{n \rightarrow \infty}\left\|x_{n+1}-x_{n}\right\|=0 .
$$


Next, we show

$$
\lim _{n \rightarrow \infty}\left\|x_{n}-T x_{n}\right\|=0
$$

We have

$$
\begin{aligned}
& \left\|x_{n+1}-T x_{n+1}\right\| \\
& \quad \leq\left\|x_{n+1}-T^{n+1} x_{n+1}\right\|+\left\|T^{n+1} x_{n+1}-T^{n+1} x_{n}\right\|+\left\|T^{n+1} x_{n}-T x_{n+1}\right\| \\
& \quad \leq\left\|x_{n+1}-T^{n+1} x_{n+1}\right\|+k_{\infty}\left\|x_{n+1}-x_{n}\right\|+k_{\infty}\left\|T^{n} x_{n}-x_{n+1}\right\| \\
& \quad \leq\left\|x_{n+1}-T^{n+1} x_{n+1}\right\|+2 k_{\infty}\left\|x_{n+1}-x_{n}\right\|+k_{\infty}\left\|T^{n} x_{n}-x_{n}\right\| .
\end{aligned}
$$

Hence, by (3.9) and (3.11), we get

$$
\lim _{n \rightarrow \infty}\left\|x_{n}-T x_{n}\right\|=0
$$

By (3.8), we have $\left\|x_{n+1}-q\right\| \leq t_{n}\left\|x_{n}-q\right\|+s_{n}$, where

$$
t_{n}=\left(1+\beta_{n}\right) k_{n}, \quad s_{n}=\beta_{n}\|u-q\|,
$$

and then we assume that $k_{n}=1+r_{n}$, so $\sum r_{n}<\infty$ for $\sum\left(k_{n}-1\right)<\infty$, now

$$
\begin{aligned}
& \left\|x_{n+m}-q\right\| \\
& \quad \leq\left(1+\beta_{n+m-1}\right)\left(1+r_{n+m-1}\right)\left\|x_{n+m-1}-q\right\|+s_{n+m-1} \\
& \quad \leq \mathrm{e}^{\beta_{n+m-1}} \mathrm{e}^{r_{n+m-1}}\left\|x_{n+m-1}-q\right\|+s_{n+m-1} \\
& \quad \leq \mathrm{e}^{\beta_{n+m-1}} \mathrm{e}^{r_{n+m-1}}\left(\mathrm{e}^{\beta_{n+m-2}} \mathrm{e}^{r_{n+m-2}}\left\|x_{n+m-2}-q\right\|+s_{n+m-2}\right)+s_{n+m-1} \\
& \quad \leq \mathrm{e}^{\beta_{n+m-1}+\beta_{n+m-2}} \mathrm{e}^{r_{n+m-1}+r_{n+m-2}}\left\|x_{n+m-2}-q\right\|+\mathrm{e}^{\beta_{n+m-1}} \mathrm{e}^{r_{n+m-1}}\left(s_{n+m-1}+s_{n+m-2}\right) \\
& \leq \cdots \\
& \quad \leq \mathrm{e}^{\sum_{i=n}^{n+m-1} \beta_{i}} \mathrm{e}^{\sum_{i=n}^{n+m-1} r_{i}}\left\|x_{n}-q\right\|+\mathrm{e}^{\sum_{i=n}^{n+m-1} \beta_{i}} \mathrm{e}^{\sum_{i=n}^{n+m-1} r_{i}} \sum_{i=n}^{n+m-1} s_{i} .
\end{aligned}
$$

By Condition (i) and the convergence of $\left\{r_{n}\right\}$, that is,

$$
\left\|x_{n+m}-q\right\| \leq M\left(\left\|x_{n}-q\right\|+\sum_{i=n}^{\infty} s_{i}\right)
$$

where $M=\mathrm{e}^{\sum_{i=n}^{n+m-1} \beta_{i}} \mathrm{e}^{\sum_{i=n}^{n+m-1} r_{i}}$, for all $m, n \geq 1$, for all $q \in \operatorname{Fix}(T)$ and for $M>0$.

Next, we prove that $\left\{x_{n}\right\}_{n=0}^{\infty}$ is a Cauchy sequence.

Since $q \in \operatorname{Fix}(T)$ arbitrarily, and $\lim _{n \rightarrow \infty}\left\|x_{n}-q\right\|$ exists, consequently, $d\left(x_{n}, F\right)$ exists by Lemma 2.5. From Lemma 2.5 and (3.12), we get

$$
\lim _{n \rightarrow \infty} f\left(d\left(x_{n}, F\right)\right) \leq \lim _{n \rightarrow \infty}\left\|x_{n}-T x_{n}\right\|=0
$$


Since $f:[0, \infty) \rightarrow[0, \infty)$ is a nondecreasing function satisfying $f(0)=0, f(r)>0$ for all $r \in(0, \infty)$, therefore, we have

$$
\lim _{n \rightarrow \infty} d\left(x_{n}, F\right)=0 .
$$

Let $\varepsilon>0$, since $\lim _{n \rightarrow \infty} d\left(x_{n}, F\right)=0$ and $\sum_{i=0}^{\infty} s_{i}<\infty$, therefore, there exists a constant $n_{0}$ such that for all $n \geq n_{0}$, we have

$$
d\left(x_{n}, F\right) \leq \frac{\varepsilon}{3 M} \quad \text { and } \quad \sum_{j=n_{0}}^{\infty} s_{j} \leq \frac{\varepsilon}{6 M}
$$

in particular,

$$
d\left(x_{n_{0}}, F\right) \leq \frac{\varepsilon}{3 M} .
$$

There must exist $p_{1} \in \operatorname{Fix}(T)$, such that

$$
d\left(x_{n_{0}}, p_{1}\right) \leq \frac{\varepsilon}{3 M} .
$$

From (3.13), it can be obtained that when $n \geq n_{0}$,

$$
\begin{aligned}
\left\|x_{n+m}-x_{n}\right\| & \leq\left\|x_{n+m}-p_{1}\right\|+\left\|x_{n}-p_{1}\right\| \\
& \leq 2 M\left(\left\|x_{n_{0}}-p_{1}\right\|+\sum_{j=n_{0}}^{n_{0}+m-1} s_{j}\right) \\
& \leq 2 M\left(\frac{\varepsilon}{3 M}+\frac{\varepsilon}{6 M}\right)=\varepsilon .
\end{aligned}
$$

This implies that $\left\{x_{n}\right\}_{n=0}^{\infty}$ is a Cauchy sequence in a closed convex subset $C$ of a Banach space $E$. Thus, it must converge to a point in $C$, let $\lim _{n \rightarrow \infty} x_{n}=p$.

For all $\epsilon>0$, as $\lim _{n \rightarrow \infty} x_{n}=p$, thus, there exists a number $n_{1}$ such that when $n_{2} \geq n_{1}$,

$$
\left\|x_{n_{2}}-p\right\| \leq \frac{\epsilon}{2+2 k_{\infty}} .
$$

In fact, $\lim _{n \rightarrow \infty} d\left(x_{n}, F\right)=0$ implies that using number $n_{2}$ above, when $n \geq n_{2}$, we have $d\left(x_{n}, F\right) \leq \frac{\epsilon}{2+6 k_{\infty}}$. In particular, $d\left(x_{n_{2}}, F\right) \leq \frac{\epsilon}{2+6 k_{\infty}}$. Thus, there must exist $\bar{p} \in \operatorname{Fix}(T)$, such that

$$
\left\|x_{n_{2}}-\bar{p}\right\|=d\left(x_{n_{2}}, \bar{p}\right)=\frac{\epsilon}{2+6 k_{\infty}} .
$$

From (3.14) and (3.15), we get

$$
\begin{aligned}
\|T p-p\| & =\left\|T p-\bar{p}+T x_{n_{2}}-\bar{p}+\bar{p}-x_{n_{2}}+x_{n_{2}}-p+\bar{p}-T x_{n_{2}}\right\| \\
& \leq\|T p-\bar{p}\|+\left\|x_{n_{2}}-\bar{p}\right\|+\left\|x_{n_{2}}-p\right\|+2\left\|T x_{n_{2}}-\bar{p}\right\| \\
& \leq k_{\infty}\|p-\bar{p}\|+\left(1+2 k_{\infty}\right)\left\|x_{n_{2}}-\bar{p}\right\|+\left\|x_{n_{2}}-p\right\|
\end{aligned}
$$




$$
\begin{aligned}
& \leq k_{\infty}\left\|x_{n_{2}}-p\right\|+k_{\infty}\left\|x_{n_{2}}-\bar{p}\right\|+\left(1+2 k_{\infty}\right)\left\|x_{n_{2}}-\bar{p}\right\|+\left\|x_{n_{2}}-p\right\| \\
& =\left(1+3 k_{\infty}\right)\left\|x_{n_{2}}-\bar{p}\right\|+\left(1+k_{\infty}\right)\left\|x_{n_{2}}-p\right\| \\
& \leq\left(1+3 k_{\infty}\right) \frac{\epsilon}{2+6 k_{\infty}}+\left(1+k_{\infty}\right) \frac{\epsilon}{2+2 k_{\infty}}=\epsilon .
\end{aligned}
$$

As $\epsilon$ is an arbitrary positive number, thus, $T p=p$, so $\left\{x_{n}\right\}_{n=0}^{\infty}$ converges strongly to a point of $T$.

Theorem 3.3 Let E be a uniformly convex Banach space, and let $T, C$ and $\left\{x_{n}\right\}_{n=0}^{\infty}$ be taken as in Theorem 3.2. Assume that E satisfies Opial's condition. If $\operatorname{Fix}(T) \neq \emptyset$, then $\left\{x_{n}\right\}_{n=0}^{\infty}$ converges weakly to a fixed point of $T$.

Proof Since $E$ is uniformly convex, from [23], $E$ is reflexive. Again by Theorem 3.2, $\left\{x_{n}\right\}$ is bounded, there exist two arbitrary subsequences $\left\{x_{n_{i}}\right\}$ and $\left\{x_{n_{j}}\right\}$ of $\left\{x_{n}\right\}$ which are weakly convergent to $x$ and $y$ in $C$, respectively. By Theorem 3.2, $\lim _{n \rightarrow \infty}\left\|x_{n}-T x_{n}\right\|=0$ and $I-T$ is demiclosed with respect to zero by Lemma 2.3. It follows that $T x=x$ and $T y=y$. Next, we prove the uniqueness. Assuming that $x \neq y$, and taking into account the fact that $\left\{x_{n_{i}}\right\}$ and $\left\{x_{n_{j}}\right\}$ are weakly convergent to $x$ and $y$, respectively, it follows from Opial's condition that

$$
\begin{aligned}
\lim _{n \rightarrow \infty}\left\|x_{n}-x\right\| & =\lim _{n_{i} \rightarrow \infty}\left\|x_{n_{i}}-x\right\|<\lim _{n_{i} \rightarrow \infty}\left\|x_{n_{i}}-y\right\|=\lim _{n \rightarrow \infty}\left\|x_{n}-y\right\| \\
& =\lim _{n_{j} \rightarrow \infty}\left\|x_{n_{j}}-y\right\|<\lim _{n_{j} \rightarrow \infty}\left\|x_{n_{j}}-x\right\|=\lim _{n \rightarrow \infty}\left\|x_{n}-x\right\| .
\end{aligned}
$$

Arriving at a contradiction, so $x=y$, then $\left\{x_{n}\right\}_{n=0}^{\infty}$ given by converges weakly to a fixed point of $T$.

\section{Strong convergence to a common fixed point of asymptotically nonexpansive semigroups and nonexpansive semigroups}

\subsection{Strong convergence theorem for nonexpansive semigroups}

Theorem 4.1 Let $C$ be a closed convex subset of a uniformly convex Banach space $E$, and let $\delta=\{T(t): t \geq 0\}$ be a nonexpansive semigroup on $C$ satisfying Condition (I) such that $\operatorname{Fix}(\&) \neq \emptyset$. Given a point $u \in C$, and given sequences $\left\{\alpha_{n}\right\}$ and $\left\{\beta_{n}\right\}$ in $(0,1)$ such that $\sum \beta_{n}<$ $\infty$ and $\left\{t_{n}\right\}$ is a positive real divergent sequence.

Define a sequence $\left\{x_{n}\right\}_{n=0}^{\infty}$ in $C$ by (1.8), then $\left\{x_{n}\right\}_{n=0}^{\infty}$ strongly converges to a common fixed point of 8 .

Proof We first show that $\left\{x_{n}\right\}$ is bounded, if we take a fixed point $q$ of $\operatorname{Fix}(\&)$.

$$
\begin{aligned}
\left\|y_{n}-q\right\| & =\left\|\alpha_{n} x_{n}+\left(1-\alpha_{n}\right) \frac{1}{t_{n}} \int_{0}^{t_{n}} T(u) x_{n} d u-q\right\| \\
& \leq \alpha_{n}\left\|x_{n}-q\right\|+\left(1-\alpha_{n}\right)\left\|\frac{1}{t_{n}} \int_{0}^{t_{n}} T(u) x_{n} d u-q\right\| \\
& \leq \alpha_{n}\left\|x_{n}-q\right\|+\left(1-\alpha_{n}\right) \frac{1}{t_{n}} \int_{0}^{t_{n}}\left\|T(u) x_{n}-q\right\| d u \\
& \leq \alpha_{n}\left\|x_{n}-q\right\|+\left(1-\alpha_{n}\right) \frac{1}{t_{n}} \int_{0}^{t_{n}}\left\|x_{n}-q\right\| d u
\end{aligned}
$$




$$
\begin{aligned}
& =\alpha_{n}\left\|x_{n}-q\right\|+\left(1-\alpha_{n}\right)\left\|x_{n}-q\right\| \\
& =\left\|x_{n}-q\right\|,
\end{aligned}
$$

we have

$$
\begin{aligned}
\left\|x_{n+1}-q\right\| & =\left\|\beta_{n} u+\left(1-\beta_{n}\right) y_{n}-q\right\| \\
& \leq \beta_{n}\|u-q\|+\left(1-\beta_{n}\right)\left\|y_{n}-q\right\| \\
& \leq \beta_{n}\|u-q\|+\left(1-\beta_{n}\right)\left\|x_{n}-q\right\| .
\end{aligned}
$$

Now, an induction yields

$$
\left\|x_{n}-q\right\| \leq \max \left\{\left\|x_{0}-q\right\|,\|u-q\|\right\}, \quad n \geq 0 .
$$

Hence, $\left\{x_{n}\right\}$ is bounded, so is $\left\{y_{n}\right\}$. We now denote $D$, the subset of $C$,

$$
D=\left\{x \in C:\|x-q\| \leq \max \left\{\left\|x_{0}-q\right\|,\|u-q\|\right\}\right\} .
$$

Also

$$
\begin{aligned}
\left\|x_{n+1}-q\right\| & =\left\|\beta_{n} u+\left(1-\beta_{n}\right) y_{n}-q\right\| \\
& \leq \beta_{n}\left\|u-y_{n}\right\|+\left\|y_{n}-q\right\| \\
& \leq \beta_{n}\|u-q\|+\beta_{n}\left\|y_{n}-q\right\|+\left\|y_{n}-q\right\| \\
& \leq\left(1+\beta_{n}\right)\left\|x_{n}-q\right\|+\beta_{n}\|u-q\| .
\end{aligned}
$$

As in the proof of Theorem 3.1, we get

$$
\lim _{n \rightarrow \infty}\left\|y_{n}-q\right\|=\lim _{n \rightarrow \infty}\left\|x_{n}-q\right\|=c
$$

Moreover, $\left\|\frac{1}{t_{n}} \int_{0}^{t_{n}} T(u) x_{n} d u-q\right\| \leq\left\|x_{n}-q\right\|$ implies that

$$
\limsup _{n \rightarrow \infty}\left\|\frac{1}{t_{n}} \int_{0}^{t_{n}} T(u) x_{n} d u-q\right\| \leq c
$$

Thus,

$$
\begin{aligned}
c & =\lim _{n \rightarrow \infty}\left\|y_{n}-q\right\|=\lim _{n \rightarrow \infty}\left\|\alpha_{n} x_{n}+\left(1-\alpha_{n}\right) \frac{1}{t_{n}} \int_{0}^{t_{n}} T(u) x_{n} d u-q\right\| \\
& =\lim _{n \rightarrow \infty}\left\|\alpha_{n}\left(x_{n}-q\right)+\left(1-\alpha_{n}\right)\left(\frac{1}{t_{n}} \int_{0}^{t_{n}} T(u) x_{n} d u-q\right)\right\|,
\end{aligned}
$$

given by Lemma 2.2 that

$$
\lim _{n \rightarrow \infty}\left\|\frac{1}{t_{n}} \int_{0}^{t_{n}} T(u) x_{n} d u-x_{n}\right\|=0 .
$$


Now,

$$
\begin{aligned}
& \left\|x_{n}-T(h) x_{n}\right\| \\
& \leq\left\|x_{n}-\frac{1}{t_{n}} \int_{0}^{t_{n}} T(u) x_{n} d u\right\|+\left\|T(h) \frac{1}{t_{n}} \int_{0}^{t_{n}} T(u) x_{n} d u-T(h) x_{n}\right\| \\
& \quad+\left\|\frac{1}{t_{n}} \int_{0}^{t_{n}} T(u) x_{n} d u-T(h) \frac{1}{t_{n}} \int_{0}^{t_{n}} T(u) x_{n} d u\right\| \\
& \leq 2\left\|x_{n}-\frac{1}{t_{n}} \int_{0}^{t_{n}} T(u) x_{n} d u\right\|+\left\|\frac{1}{t_{n}} \int_{0}^{t_{n}} T(u) x_{n} d u-T(h) \frac{1}{t_{n}} \int_{0}^{t_{n}} T(u) x_{n} d u\right\|,
\end{aligned}
$$

by Lemma 2.6 , we get

$$
\lim _{t \rightarrow \infty} \sup _{x_{n} \in D}\left\|\frac{1}{t_{n}} \int_{0}^{t_{n}} T(u) x_{n} d u-T(h) \frac{1}{t_{n}} \int_{0}^{t_{n}} T(u) x_{n} d u\right\|=0
$$

for every $h \in[0, \infty)$. From (4.1), we obtain

$$
\lim _{n \rightarrow \infty} \sup _{x_{n} \in D}\left\|x_{n}-T(h) x_{n}\right\|=0
$$

for every $h \in[0, \infty)$.

Since $\{T(t): t \geq 0\}$ is a nonexpansive semigroup, and $\left\{t_{n}\right\}$ is a positive real divergent sequence, then, for all $h \geq 0$ and the bounded closed convex subset $D$ of $C$ containing $\left\{x_{n}\right\}$,

$$
\lim _{n \rightarrow \infty}\left\|x_{n}-T(h) x_{n}\right\| \leq \lim _{n \rightarrow \infty} \sup _{x_{n} \in D}\left\|x_{n}-T(h) x_{n}\right\|=0 .
$$

As in the proof of Theorem 3.1, we have $x_{n} \rightarrow p(p \in \operatorname{Fix}(8))$.

\subsection{Strong convergence theorem for asymptotically nonexpansive semigroups}

In this part, assume that $\delta=\{T(t): t \geq 0\}$ is an asymptotically nonexpansive semigroup defined on a nonempty closed convex subset $C$ of a Banach space $E$. Recall that we use $L_{t}$ to denote Lipschitzian constant of the mapping $T(t)$, and assume that $L_{t}$ is bounded and measurable so that the integral $\int_{0}^{t} L_{s} d s$ exists for all $t>0$. Recall also that $L_{t} \geq 1$ for all $t>0, L_{t}$ is nonincreasing in $t$, and $\lim _{t \rightarrow \infty} L_{t}=1$. In the rest of this part, we put $\widetilde{L}_{t}=$ $\max \left\{\sup _{s \geq t} L_{s}, 1\right\}<\infty$ for each $t>0$.

Theorem 4.2 Let $C$ be a closed convex subset of a uniformly convex Banach space E, and let $\delta=\{T(t): t \geq 0\}$ be an asymptotically nonexpansive semigroup on $C$ satisfying Condition (I) such that $\operatorname{Fix}(\wp) \neq \emptyset$. Given a point $u \in C$, and given sequences $\left\{\alpha_{n}\right\}$ and $\left\{\beta_{n}\right\}$ in $(0,1)$,

$\left\{t_{n}\right\}$ is a positive real divergent sequence, the following conditions are satisfied:

(i) $\sum \beta_{n}<\infty$;

(ii) $\sum\left(\widetilde{L}_{t}-1\right)<\infty$.

Define a sequence $\left\{x_{n}\right\}_{n=0}^{\infty}$ in C by (1.8), then $\left\{x_{n}\right\}_{n=0}^{\infty}$ strongly converges to a common fixed point of 8 . 
Proof We first show that $\left\{x_{n}\right\}$ is bounded if we take a fixed point $q$ of Fix $(\&)$.

$$
\begin{aligned}
\left\|y_{n}-q\right\| & =\left\|\alpha_{n} x_{n}+\left(1-\alpha_{n}\right) \frac{1}{t_{n}} \int_{0}^{t_{n}} T(u) x_{n} d u-q\right\| \\
& \leq \alpha_{n}\left\|x_{n}-q\right\|+\left(1-\alpha_{n}\right)\left\|\frac{1}{t_{n}} \int_{0}^{t_{n}} T(u) x_{n} d u-q\right\| \\
& \leq \alpha_{n}\left\|x_{n}-q\right\|+\left(1-\alpha_{n}\right) \frac{1}{t_{n}} \int_{0}^{t_{n}}\left\|T(u) x_{n}-q\right\| d u \\
& \leq \alpha_{n}\left\|x_{n}-q\right\|+\left(1-\alpha_{n}\right) \frac{1}{t_{n}} \int_{0}^{t_{n}} L_{u} d u\left\|x_{n}-q\right\| \\
& \leq \frac{1}{t_{n}} \int_{0}^{t_{n}} L_{u} d u\left\|x_{n}-q\right\| \leq \widetilde{L}_{t}\left\|x_{n}-q\right\|,
\end{aligned}
$$

we have

$$
\begin{aligned}
\left\|x_{n+1}-q\right\| & =\left\|\beta_{n} u+\left(1-\beta_{n}\right) y_{n}-q\right\| \\
& \leq \beta_{n}\|u-q\|+\left(1-\beta_{n}\right)\left\|y_{n}-q\right\| \\
& \leq \beta_{n}\|u-q\|+\widetilde{L}_{t}\left(1-\beta_{n}\right)\left\|x_{n}-q\right\| \\
& \leq \widetilde{L}_{t} \max \left\{\|u-q\|,\left\|x_{n}-q\right\|\right\} .
\end{aligned}
$$

Now, an induction yields

$$
\left\|x_{n}-q\right\| \leq \widetilde{L}_{t} \max \left\{\left\|x_{0}-q\right\|,\|u-q\|\right\}, \quad n \geq 0 .
$$

Since $\widetilde{L}_{t}=\max \left\{\sup _{s \geq t} L_{s}, 1\right\}<\infty$, hence, $\left\{x_{n}\right\}_{n=0}^{\infty}$ is bounded, so is $\left\{y_{n}\right\}$. We now denote $D$, the subset of $C$

$$
D=\left\{x \in C:\|x-q\| \leq \widetilde{L}_{t} \max \left\{\left\|x_{0}-q\right\|,\|u-q\|\right\}\right\} .
$$

Also

$$
\begin{aligned}
\left\|x_{n+1}-q\right\| & =\left\|\beta_{n} u+\left(1-\beta_{n}\right) y_{n}-q\right\| \\
& \leq \beta_{n}\left\|u-y_{n}\right\|+\left\|y_{n}-q\right\| \\
& \leq \beta_{n}\|u-q\|+\beta_{n}\left\|y_{n}-q\right\|+\left\|y_{n}-q\right\| \\
& \leq\left[1+\left(\beta_{n} \widetilde{L}_{t}+\widetilde{L}_{t}-1\right)\right]\left\|x_{n}-q\right\|+\beta_{n}\|u-q\| .
\end{aligned}
$$

Thus, by Condition (i), (ii) and following from Lemma 2.1, there exists $\lim _{n \rightarrow \infty}\left\|x_{n}-q\right\|$. As in the proof of Theorem 3.2, we get

$$
\lim _{n \rightarrow \infty}\left\|y_{n}-q\right\|=\lim _{n \rightarrow \infty}\left\|x_{n}-q\right\|=c
$$

Moreover, $\left\|\frac{1}{t_{n}} \int_{0}^{t_{n}} T(u) x_{n} d u-q\right\| \leq \widetilde{L}_{t}\left\|x_{n}-q\right\|$, which implies that

$$
\limsup _{n \rightarrow \infty}\left\|\frac{1}{t_{n}} \int_{0}^{t_{n}} T(u) x_{n} d u-q\right\| \leq c .
$$


Thus,

$$
\begin{aligned}
c & =\lim _{n \rightarrow \infty}\left\|y_{n}-q\right\|=\lim _{n \rightarrow \infty}\left\|\alpha_{n} x_{n}+\left(1-\alpha_{n}\right) \frac{1}{t_{n}} \int_{0}^{t_{n}} T(u) x_{n} d u-q\right\| \\
& =\lim _{n \rightarrow \infty}\left\|\alpha_{n}\left(x_{n}-q\right)+\left(1-\alpha_{n}\right)\left(\frac{1}{t_{n}} \int_{0}^{t_{n}} T(u) x_{n} d u-q\right)\right\|,
\end{aligned}
$$

given by Lemma 2.2,

$$
\lim _{n \rightarrow \infty}\left\|\frac{1}{t_{n}} \int_{0}^{t_{n}} T(u) x_{n} d u-x_{n}\right\|=0 .
$$

Now,

$$
\begin{aligned}
& \left\|x_{n}-T(h) x_{n}\right\| \\
& \leq\left\|x_{n}-\frac{1}{t_{n}} \int_{0}^{t_{n}} T(u) x_{n} d u\right\|+\left\|T(h) \frac{1}{t_{n}} \int_{0}^{t_{n}} T(u) x_{n} d u-T(h) x_{n}\right\| \\
& \quad+\left\|\frac{1}{t_{n}} \int_{0}^{t_{n}} T(u) x_{n} d u-T(h) \frac{1}{t_{n}} \int_{0}^{t_{n}} T(u) x_{n} d u\right\| \\
& \leq \\
& \quad\left(1+\widetilde{L}_{t}\right)\left\|x_{n}-\frac{1}{t_{n}} \int_{0}^{t_{n}} T(u) x_{n} d u\right\| \\
& \quad+\left\|\frac{1}{t_{n}} \int_{0}^{t_{n}} T(u) x_{n} d u-T(h) \frac{1}{t_{n}} \int_{0}^{t_{n}} T(u) x_{n} d u\right\|,
\end{aligned}
$$

by Lemma 2.6 , we get

$$
\lim _{t \rightarrow \infty} \sup _{x_{n} \in D}\left\|\frac{1}{t_{n}} \int_{0}^{t_{n}} T(u) x_{n} d u-T(h) \frac{1}{t_{n}} \int_{0}^{t_{n}} T(u) x_{n} d u\right\|=0
$$

for every $h \in[0, \infty)$. From (4.2), we obtain

$$
\lim _{n \rightarrow \infty} \sup _{x_{n} \in D}\left\|x_{n}-T(h) x_{n}\right\|=0
$$

for every $h \in[0, \infty)$.

Since $\{T(t): t \geq 0\}$ is asymptotically nonexpansive semigroup, and $\left\{t_{n}\right\}$ is a positive real divergent sequence, then, for all $h \geq 0$, and for the bounded closed convex subset $D$ of $C$ containing $\left\{x_{n}\right\}$,

$$
\lim _{n \rightarrow \infty}\left\|x_{n}-T(h) x_{n}\right\| \leq \lim _{n \rightarrow \infty} \sup _{x_{n} \in D}\left\|x_{n}-T(h) x_{n}\right\|=0 .
$$

As in the proof of Theorem 3.2, we have $x_{n} \rightarrow p(p \in \operatorname{Fix}(8))$.

\section{Application}

Let $E$ be a real Banach space. Recall that an operator (possibly multivalued) $A$ with domain $D(A)$ and range $R(A)$ in $E$ is said to be accretive if, for each $x_{i} \in D(A)$ and $y_{i} \in A x_{i}(i=1,2)$, there exists a $j\left(x_{1}-x_{2}\right) \in J\left(x_{1}-x_{2}\right)$ such that

$$
\left\langle y_{1}-y_{2}, j\left(x_{1}-x_{2}\right)\right\rangle \geq 0
$$


where $J$ is the normalized duality map from $E$ to the dual space $E^{*}$ given by

$$
J(x)=\left\{x^{*} \in E^{*}:\left\langle x, x^{*}\right\rangle=\|x\|^{2}=\left\|x^{*}\right\|^{2}\right\}, \quad x \in E .
$$

An accretive operator $A$ is $m$-accretive if $R(I+r A)=E$ for all $r>0$. Denote the zero set of $A$ by

$$
F:=A^{-1}(0)=\{z \in D(A): 0 \in A z\} .
$$

For an $m$-accretive operator $A$ with $F \neq \emptyset$ and $C=\overline{D(A)}$ convex, the problem of finding a zero of $A$, i.e.,

find $z \in C$ such that $0 \in A z$,

has extensively been investigated due to its applications in related problems such as minimization problems, variational inequality problems and nonlinear evolution equations.

It is known that the resolvent of $A$, defined by

$$
J_{r}=(I+r A)^{-1},
$$

for $r>0$, is a nonexpansive mapping from $E$ to $C$, and it is straightforward to see that $F$ coincides with the fixed point set of $J_{r}$ for any $r>0$. Therefore, (5.1) is equivalent to the fixed point problem $z=J_{r} z$. Then an interesting approach to solving this problem is via iterative methods for nonexpansive mappings. We need the resolvent identity [23].

Theorem 5.1 Let $E$ be a uniformly convex Banach space, and let $A$ be an m-accretive operator in $E$ such that $A^{-1}(0) \neq \emptyset, J_{r}: E \rightarrow E$ is nonexpansive for all $r>0$ satisfying Condition (I). Given a point $u \in E$, and given sequences $\left\{\alpha_{n}\right\}$ and $\left\{\beta_{n}\right\}$ in $(0,1)$, the following conditions are satisfied:

(i) $\sum \beta_{n}<\infty$;

(ii) $r_{n} \geq \varepsilon$ for some $\varepsilon>0$ and for all $n \geq 1$.

Define a sequence $\left\{x_{n}\right\}_{n=0}^{\infty}$ by (1.9), then $\left\{x_{n}\right\}_{n=0}^{\infty}$ strongly converges to a zero of $A$.

Proof Take any arbitrary $q \in F=A^{-1}(0)$, it follows from Lemma 2.1 that $\lim _{n \rightarrow \infty}\left\|x_{n}-q\right\|$ exists. From Lemma 2.2, it can be shown that $\lim _{n \rightarrow \infty}\left\|J_{r_{n}} x_{n}-x_{n}\right\|=0$. Since $J_{r}: E \rightarrow$ $E$ is nonexpansive for all $r>0$ satisfying Condition (I), it follows from Lemma 2.7 that $\lim _{n \rightarrow \infty}\left\|J_{r} x_{n}-x_{n}\right\|=0$. Therefore, all the conditions in Theorem 3.1 are satisfied. The conclusion of Theorem 5.1 can be obtained from Theorem 3.1 immediately.

Theorem 5.2 Let $E$ be a uniformly convex Banach space, and let $A$ be an m-accretive operator in $E$ such that $\operatorname{Fix}\left(J_{r_{1}}\right) \cap \operatorname{Fix}\left(J_{r_{2}}\right)=A^{-1}(0) \neq \emptyset, J_{r_{i}}: E \rightarrow E$ is nonexpansive for all $r_{i}>0(i=1,2)$ satisfying Condition (I). Given sequences $\left\{\alpha_{n}\right\}$ and $\left\{\beta_{n}\right\}$ in $(0,1)$, the following conditions are satisfied:

(i) $\sum \beta_{n}<\infty$;

(ii) $r_{i, n} \geq \varepsilon$ for some $\varepsilon>0$ and for all $n \geq 1$.

Define a sequence $\left\{x_{n}\right\}_{n=0}^{\infty}$ by (1.10), then $\left\{x_{n}\right\}_{n=0}^{\infty}$ strongly converges to a zero of $A$. 
Proof Only a sketch of the proof is given here. Take any arbitrary $q \in \operatorname{Fix}\left(J_{r_{1}}\right) \cap \operatorname{Fix}\left(J_{r_{2}}\right)=$ $A^{-1}(0)$, it follows from Lemma 2.1 that $\lim _{n \rightarrow \infty}\left\|x_{n}-q\right\|$ exists. From Lemma 2.2 , it can be shown that $\lim _{n \rightarrow \infty}\left\|J_{r_{i, n}} x_{n}-x_{n}\right\|=0(i=1,2)$. Since $J_{r_{i}}: E \rightarrow E$ is nonexpansive for all $r_{i}>0$ satisfying Condition (I), it follows from Lemma 2.7 that $\lim _{n \rightarrow \infty}\left\|J_{r_{1}} x_{n}-x_{n}\right\|=$ $\lim _{n \rightarrow \infty}\left\|J_{r_{2}} x_{n}-x_{n}\right\|=0$. Therefore, all the conditions in Theorem 3.1 are satisfied. The conclusion of Theorem 5.2 can be obtained from Theorem 3.1 immediately.

\section{Competing interests}

The authors declare that they have no competing interests.

\section{Authors' contributions}

All authors contributed equally and significantly in writing this paper. All authors read and approved the final manuscript.

\section{Acknowledgements}

This work was supported by the National Natural Science Foundation of China (Grant No. 11171046).

Received: 20 June 2013 Accepted: 30 September 2013 Published: 08 Nov 2013

\section{References}

1. Browder, FE: Fixed point theorems for noncompact mappings in Hilbert space. Proc. Natl. Acad. Sci. USA 53, 1272-1276 (1965)

2. Goebel, K, Kirk, WA: A fixed point theorem for asymptotically nonexpansive mappings. Proc. Am. Math. Soc. 35, 171-174 (1972)

3. Shioji, N, Takahashi, W: Strong convergence theorems for continuous semigroups in Banach spaces. Math. Jpn. 50, 57-66 (1999)

4. Deutsch, F, Yamada, I: Minimizing certain convex functions over the intersection of the fixed point sets of nonexpansive mappings. Numer. Funct. Anal. Optim. 19, 33-56 (1998)

5. Xu, HK: Iterative algorithms for nonlinear operators. J. Lond. Math. Soc. 66, 240-256 (2002)

6. Youla, D: Mathematical theory of image restoration by the method of convex projection. In: Stark, $\mathrm{H}$ (ed.) Image Recovery: Theory and Applications, pp. 29-77. Academic Press, Orlando (1987)

7. Byrne, $C:$ A unified treatment of some iterative algorithms in signal processing and image construction. Inverse Probl. 20, 103-120 (2004)

8. Combettes, PL: The convex feasibility problem in image recovery. In: Advances in Imaging and Electron Physics, vol. 95, pp. 155-270. Academic Press, New York (1996)

9. Kitahara, S, Takahashi, W: Image recovery by convex combinations of sunny nonexpansive retractions. Topol. Methods Nonlinear Anal. 2, 333-342 (1993)

10. Mann, WR: Mean value methods on iteration. Proc. Am. Math. Soc. 4, 506-510 (1953)

11. Ishikawa, S: Fixed points by a new iteration method. Proc. Am. Math. Soc. 44, 147-150 (1974)

12. Halpern, B: Fixed points of nonexpanding maps. Bull. Am. Math. Soc. 73, 957-961 (1967)

13. Reich, S: Weak convergence theorems for nonexpansive mappings in Banach spaces. J. Math. Anal. Appl. 67, 274-276 (1979)

14. Nakajo, K, Takahashi, W: Strong convergence theorems for nonexpansive mappings and nonexpansive semigroups. J. Math. Anal. Appl. 279, 372-379 (2003)

15. Kim, TH, Xu, HK: Strong convergence of modified Mann iterations for asymptotically nonexpansive mappings and semigroups. Nonlinear Anal. 64, 1140-1152 (2006)

16. Kim, TH, Xu, HK: Strong convergence of modified Mann iterations. Nonlinear Anal. 61, 51-60 (2005)

17. Nammanee, K, Noor, MA, Suantai, S: Convergence criteria of modified Noor iterations with errors for asymptotically nonexpansive mappings. J. Math. Anal. Appl. 314, 320-334 (2006)

18. Schu, J: Weak and strong convergence to fixed points of asymptotically nonexpansive mappings. Bull. Aust. Math Soc. 43, 153-159 (1991)

19. Cho, YJ, Zhou, HY, Guo, GT: Weak and strong convergence theorems for three-step iterations with errors for asymptotically nonexpansive mappings. Comput. Math. Appl. 47, 707-717 (2004)

20. Opial, Z: Weak convergence of the sequence of successive approximations for nonexpansive mappings. Bull. Am. Math. Soc. 73, 591-597 (1967)

21. Senter, HF, Dotson, WG Jr.: Approximating fixed points of non-expansive mappings. Proc. Am. Math. Soc. 44, 375-380 (1974)

22. Chen, R, Song, Y: Convergence to common fixed point of nonexpansive semigroups. J. Math. Anal. Appl. 200, 566-575 (2007)

23. Barbu, V: Nonlinear Semi-Groups and Differential Equations in Banach Space. Noordhoff, Leiden (1976)

10.1186/1687-1812-2013-282

Cite this article as: Chen and Wu: Convergence theorems of modified Mann iterations. Fixed Point Theory and Applications 2013, 2013:282 